\title{
Correlation between serum neopterin level and carotid artery intima- media thickness in type 2 diabetic patients
}

\author{
Mashahet MA ${ }^{(1)}$, Abouelseoud $\mathrm{SN}^{(2)}$, Zaki OMA ${ }^{(3)}$ and Mahmoud \\ $\mathrm{MAM}^{(4)}$.
}

(1) professor and head of department of internal medicine department, Faculty of Medicine Fayoum University

(2)Lecturer of internal medicine , Faculty of Medicine Fayoum university.

(3)lecturer of clinical and chemical pathology, Faculty of

Medicine Fayoum University.

(4) Department of internal medicine, Fayoum general hospital.

\begin{abstract}
Background:

Atherosclerosis is currently viewed as an immune-mediate inflammatory disease of the arterial wall, macrophages play an important role in atherogenesis.Inflammatory mechanisms with atherosclerotic plaque formation can be triggered,maintained and enhanced by multiple factors such as activated macrophages that induce synthesis of neopterin.
\end{abstract}

\section{The aim of the study:}

To evaluate the association between serum neopterin level and carotid atherosclerosis in type 2 diabetic patient and to highlight the role of inflammation in atherosclerosis and atherosclerotic cardiovascular disease.

\section{Methods:}

This is a prospective study include (96) subjects, they were divided into three groups: group A (32) T2DM patients without cardiovascular complications, group B (32) T2DM patients with cardiovascular complications and group C (32) healthy control subjects.All Subjects in this study were subjected to full clinical examination and laboratory investigations as FBS,2hrsPP,HBA1c,lipid profile. measurement of serum neopterin level, hs$\mathrm{CRP}$ and carotid duplex to measure carotid artery intima-media thickness.

\section{Results:}

Serum neopterin shows higher values in group B patients with a statistical positive correlation between serum neopterin and hs-CRP in group B p-values 0.041, also serum neopterin shows higher values in patients with echolucent plaques in group B. A statistical positive correlation between serum neopterin and CA-IMT.was found P.value $<0.0001$ in diabetic patients groups $(\mathrm{A}+\mathrm{B})$,also, A statistical positive correlation between serum neopterin and hs-CRP. was found P.value : $<0.0001$ in diabetic patients groups $(\mathrm{A}+\mathrm{B})$.

\section{Conclusions:}

Serum neopterin is a marker of both macrophage activation and redox system activation and could be used as a potential biomarker in risk stratification while assessing and managing atherosclerotic cardiovascular diseases. 
Keywords: Neopterin, Inflammation,

\section{INTRODUCTION}

Patients with diabetes mellitus (DM) and associated microvascular complications appear particularly at higher risk of accelerated atherosclerosis, which ultimately culminates in cerebrovascular and cardiovascular events, and premature death (Kalofoutis et al., 2007). Microvessels are the basic functional unit of the cardiovascular system comprising of arterioles, capillaries, and venules (Orasanu et al., 2009).

Atherosclerosis is the focal expression of a systemic disease affecting medium- and large-sized arteries, in which traditional cardiovascular risk factor and immune factors play a key role. It is well accepted that circulating biomarkers, involving C-reactive protein and interleukin6, reliably predict major cardiovascular events, including myocardial infarction or death (Ammirati et al., 2015).

\section{Aim of the Work}

To study correlation between serum level of neopterin and carotid intima media thickness in type 2 diabetic patients and to focus on the role of inflammation and
Atherosclerosis, Diabetes mellitus

Inflammatory cytokines enter the circulation in soluble form. The concept of inflammation involvement in atherosclerosis has validated the adoption of inflammatory biomarkers for cerebrovascular risk stratification and prediction (Packard et al., 2008).

Neopterin is a pteridine released from activated monocytes and macrophages upon stimulation with the Th1-type cytokine interferon- $\gamma($ IFN- $\gamma)$, and reflects the cellular immune response. In addition, neopterin is a sensitive marker of reactive oxygen species generation in macrophages (Bjørnestad et al., 2017). Neopterin was used a novel marker for carotid artery stenosis in community subjects in 2015 (Liao et al., 2015).

Its plasma concentration is elevated in patients with coronary artery disease (CAD), with neopterin abundantly expressed within atherosclerotic lesions in human coronary and carotid arteries and aorta (Shirai et al., 2018).

immune system in atherosclerotic cardiovascular disease

\section{Patients and methods}

This is a prospective study will include (97) subjects, they were divided into three groups: 
Group A: (3r) patients with type 2 diabetes without cardiovascular and cerebrovascular complications. (Diabetic patients of shorter duration less than 5 years in whom carotid artery atherosclerotic plaques is less likely to be present)

Group B: $\left(3^{r}\right)$ patients with type 2 diabetes with cardiovascular and cerebrovascular complications. (Diabetic patients of longer duration more than 5 years in whom carotid artery atherosclerotic plaques is more likely to be present)

Group C: ( $(r)$ healthy matched nondiabetic individuals as a control.

The patients were collected from the Diabetes Clinic of Fayoum University
Hospital and from al-Fayoum general hospital their ages are ranging from 18 to 75 years old.

In this study, we measured the serum level of neopterin in type 2 diabetic patients with and without diabetic macrovascular complications and compare them both to the control group.

In addition, we tried to find a correlation between neopterin level and carotid artery atherosclerosis detected by measuring carotid artery intima media thickness via carotid Doppler ultrasonography.

\section{RESULTS}

1- There was a statistical positive correlation between serum neopterin and hs-CRP in Group B, p-values $\mathbf{0 . 0 4 1}$. Also, there was positive correlation between serum neopterin and hsCRP but of not statistically significance, p-values 0.127 and 0.598 in Group A and C, respectively. 


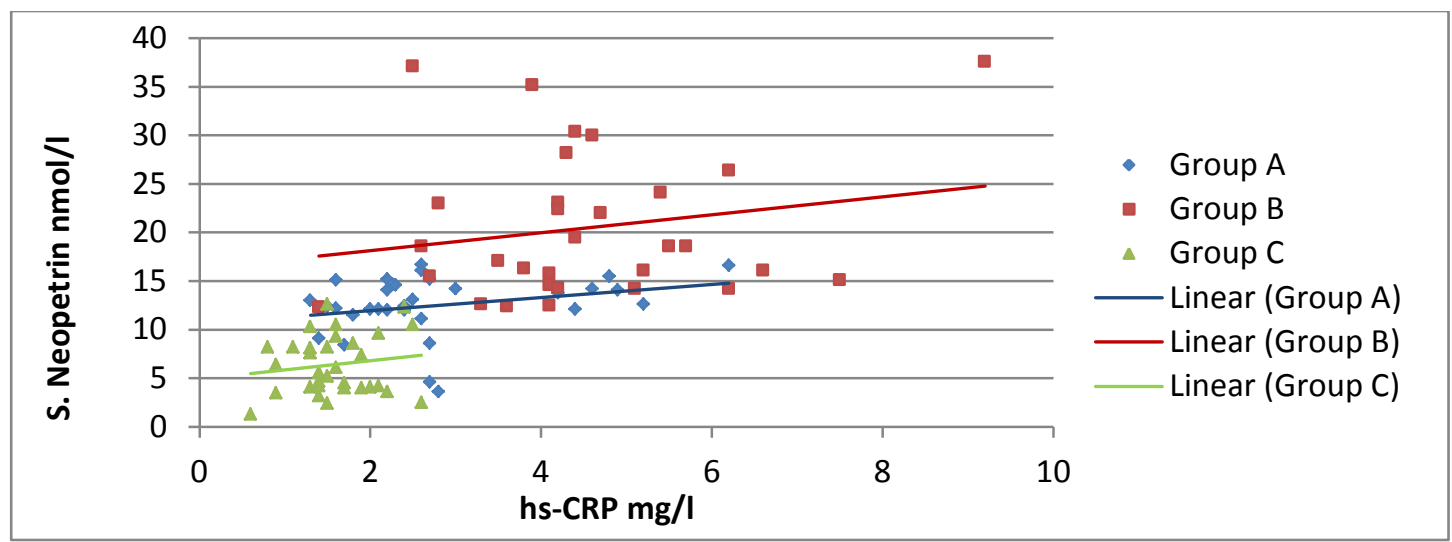

2- although there were positive correlation of serum nerpterin with CA.IMT but they were not statistically significant in all groups, p-values $\mathbf{0 . 2 1 8}, \mathbf{0 . 2 9 9}$ and $\mathbf{0 . 4 1 4}$ in group A. B and $\mathrm{C}$, respectively.

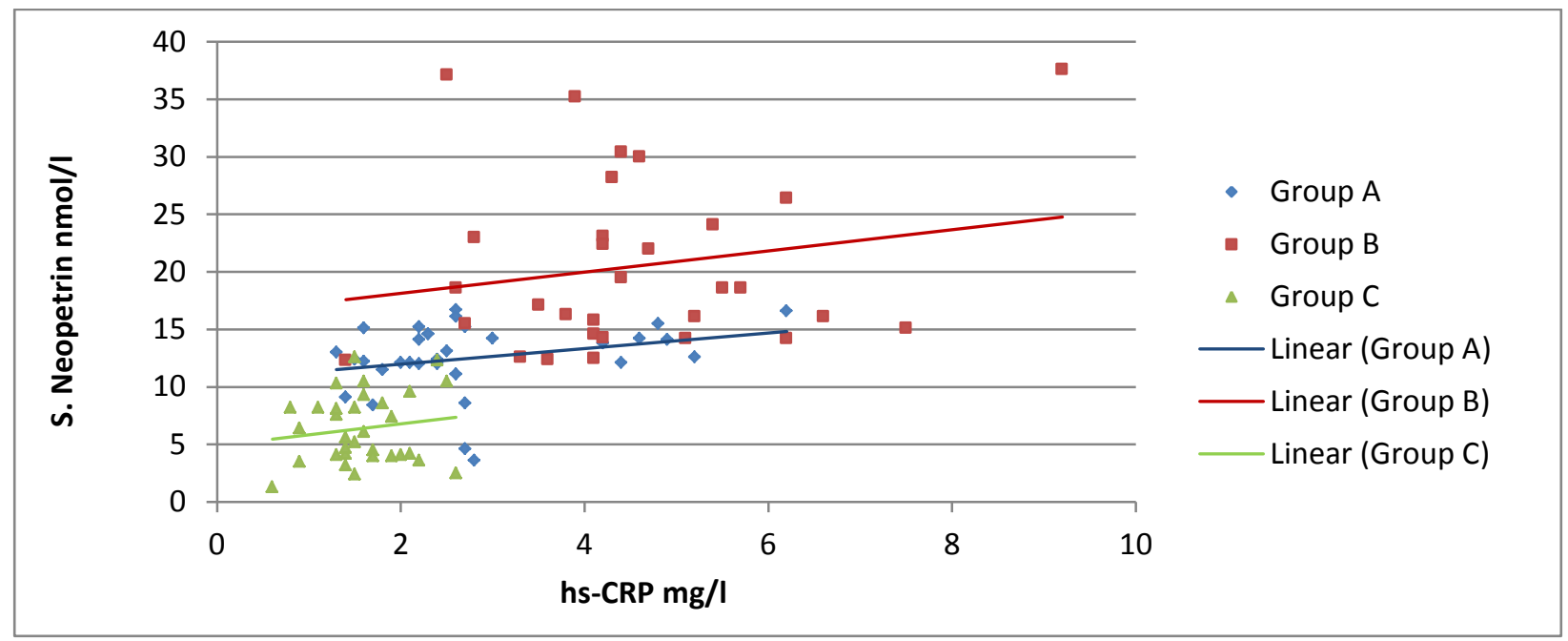

3-the mean serum neopterin level was $12.51 \pm 3.03 \mathrm{nmol} / \mathrm{l}$ with minimum value 3.6 nmol/l and maximum $22 \mathrm{nmol} / \mathrm{l}$ in Group A patients, the mean serum neopterin level $20.37 \pm$ $7.35 \mathrm{nmol} / \mathrm{l}$ with minimum value of $12.3 \mathrm{nmol} / \mathrm{l}$ and maximum one of $37.6 \mathrm{nmol} / \mathrm{l}$ in Group B patients. The mean serum neopterin level was $6.41 \pm 3.03 \mathrm{nmol} / 1$ with minimum value of 1.3 $\mathrm{nmol} / \mathrm{l}$ and maximum $12.6 \mathrm{nmol} / \mathrm{l}$ in Group C. Neopterin levels showed highly significant statistical difference between the three groups. 


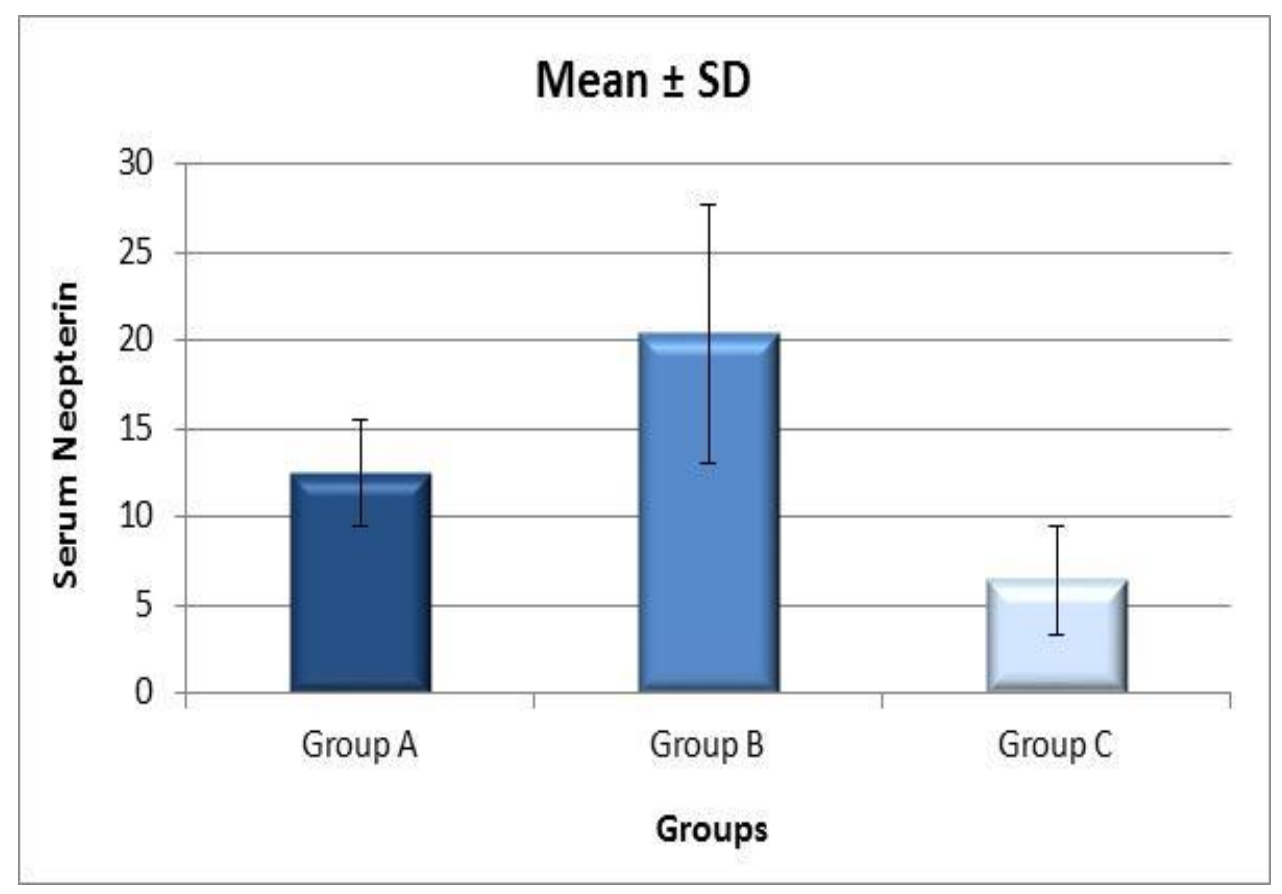

4- It was clear that, among Group (B) patients, (4) has no plaque, (9) patients have echogenic plaque and (19) patients have echolucent plaque. Neopterin level in the no plaque group, echogenic plaque group and echolucent plaque group were $(9.79 \pm 4.43) \mathrm{nmol} / \mathrm{L},(14.61 \pm$ $1.39(\mathrm{nmol} / \mathrm{L}$ and $(24.20 \pm 7.23) \mathrm{nmol} / \mathrm{L}$, respectively . There were significant differences between no plaque group and echogenic plaque group, no plaque group and echolucent plaque group.

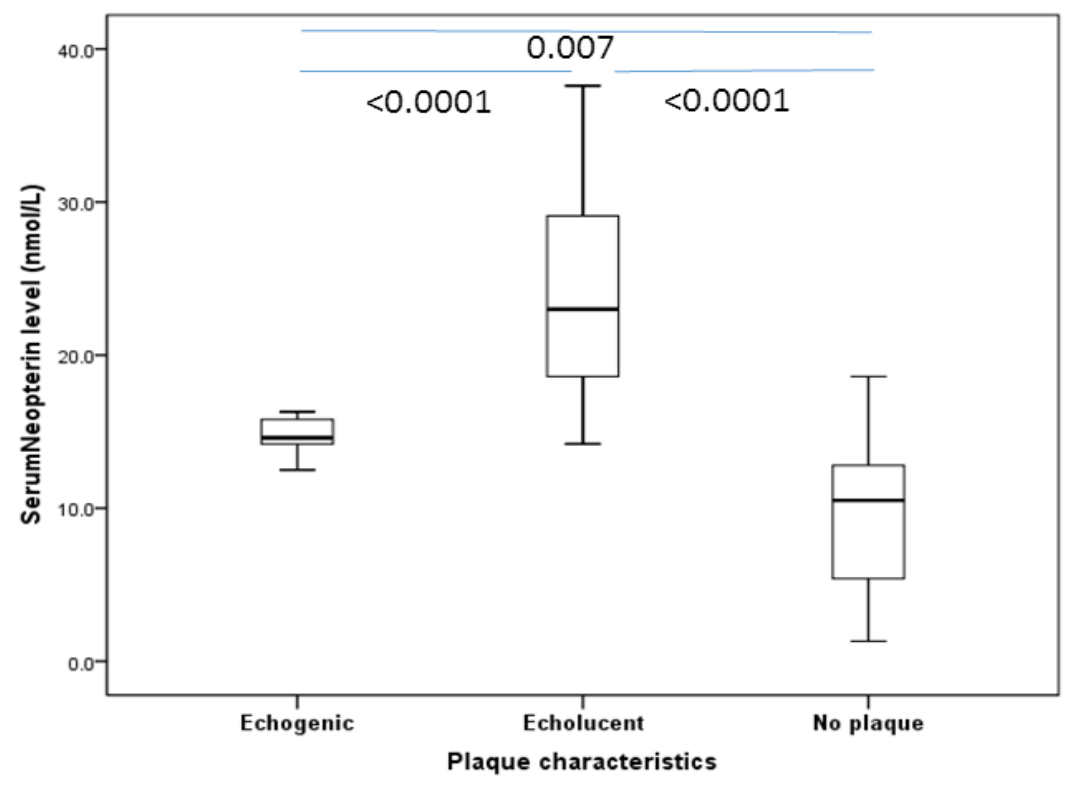




\section{DISCUSSION}

Atherosclerosis is a chronic inflammatory disease characterised by intimal thickening and deposition of macrophages in the artery wall. The inflammatory nature of the disease is highlighted by the correlation between plasma neopterin levels and increasing severity of cardiovascular disease (Lin et al., 2012).

Neopterin is a highly fluorescent pterin compound, whose measurement in urine and plasma has been extensively used as a marker of inflammation in a wide number of inflammatory conditions including vascular disease (Gieseg et al., 2008).

Since neopterin is induced through IFN- $\gamma$ secretion, its concentration might be considered as an indicator of systemic immune activation, reflecting in particular the activity of both innate myeloid cells (monocytes and macrophages) and of lymphoid cells ( $\mathrm{T}$ and NK cells). Also, neopterin generation has been shown to correlate with reactive oxygen species (ROS) production suggesting neopterin as an indicator for oxidative stress (Larsen et al., 2017).

Neopterin is also known as an activation marker for monocytes/macrophages (Fuchs et al., 1993). The specific receptors for neopterin have yet to be identified. Previous studies have reported that neopterin has antioxidant properties (Kojima et al., 1992). Its plasma concentration is elevated in patients with coronary artery disease CAD) (Murr et al., 2014) with neopterin abundantly expressed within atherosclerotic lesions in human coronary and carotid arteries and aorta. (van Dijk et al., 2016).

Regarding hsCRP, in this current high sensitive c-reactive protein was high in grope $B$ diabetic patients with cardiovascular complications of value $4.53 \pm$ $1.5 \mathrm{mg} / \mathrm{L}$ compared to $2.80 \pm 1.24 \mathrm{mg} / \mathrm{L}$ in group A diabetic patient without cardiovascular complications and of control group C $1.59 \pm 0.48 \mathrm{mg} / \mathrm{L}$, there was a significant correlations between hsCRP and carotid intima- media thickness $\mathrm{p}$. value in group A,B and $\mathrm{C}$ was $<\mathbf{0 . 0 0 0 1 , 0 . 0 0 4}$ and 0.945 respectively. This observation is in agreement with Parildar et al.,(2013) There was a positive, signifcant correlation between left, right,maximum CIMT and blood glucose, HbA1c, BMI and hs-CRP, and results of Kablak-Ziembicka et al., (2011) who showed that carotid intimamedia thickness, hs-CRP and TNF-are independently associated with cardiovascular event risk in patients with atherosclerotic occlusive disease, also results of Abdushi et al., (2013) stated that increased carotid intima-media thickness associated with high hs-Crp levels is a predictor of unstable coronary artery disease 
Regarding carotid plaque echogenicity, in the current study among group (B) patients, (4) have no plaque, (9) patients have echogenic plaque and (19) patients have echolucent plaque. Neopterin level in the no plaque group, echogenic plaque group and echolucent plaque group were $(9.79 \pm 4.43) \mathrm{nmol} / \mathrm{L}, \quad(14.61 \pm$ $1.39(\mathrm{nmol} / \mathrm{L}$ and $(24.20 \pm 7.23) \mathrm{nmol} / \mathrm{L}$, respectively.

There were significant differences between no plaque group and echogenic plaque group, no plaque group and echolucent plaque group. Serum neopterin was higher in echolucent plaque than echogenic plaque this observation is in agreement with Liao et al., (2015) demonstrate that circulating neopterin level is increased in subclinical carotid atherosclerosis population with CAS and reinforce the key roles of inflammatory response in the pathogenesis.

Similar findings were observed in the results of Sugioka et al., (2010) who found that elevated levels of neopterin are associated with carotid plaques with complex morphology in patients with stable angina pectoris.
Concerning serum neopterin, in this study serum neopterin level was higher in group B diabetic with cardiovascular complications $20.37 \pm 7.35 \mathrm{nmol} / \mathrm{l}$ compared to $12.51 \pm 3.03 \mathrm{nmol} / \mathrm{l}$ in group A diabetic patients without cardiovascular complications and to control group C $6.41 \pm$ $3.03 \mathrm{nmol} / \mathrm{l}$ with significant statistical difference between the 3 groups. serum neopterin shows higher level in patients with cardiovascular events in the form of ACS and ischemic stroke,

This observation is in agreement with Liu and Li, (2013) which revealed that neopterin concentrations of the AMI and UAP groups were signifcantly higher than those of the SAP and control groups and results of Zeng et al.,(2016) who showed that the neopterin serum levels were significantly $(\mathrm{P}<0.0001)$ higher in acute ischemic stroke patients as compared to normal controls.

Also we found in this study positive correlation between serum neopterin and cIMT in diabetic groups as compared by control group ,p value $<0.0001$ and this matches with results of Liao et al.,(2015) which conclude that circulating neopterin level is increased in subclinical carotid atherosclerosis population with carotid 
artery stenosis and reinforce the key roles of inflammatory response in the pathogenesis.

In this study there was a statistically significant correlation between serum neopterin and hsCRP in diabetic groups versus control group $\mathrm{P}$. value of $\mathbf{0 . 0 0 0 1}$ versus 0.598 also in diabetic group B with cardiovascular complications, $\mathrm{p}$ value 0.041 , these results were in agreement with Elayalwar and Lakshmipathi, (2016) who proved that high levels of serum neopterin is correlated significantly to high sensitive CRP. $(\mathrm{p}<0.000<0.05)$

\section{Summary and conclusion}

Recently inflammation becomes a target in the management of atherosclerotic cardiovascular diseases based on the fact that atherosclerosis is a progressive chronic inflammatory process and in particular in patients with residual cardiovascular risk.

Early detection of vulnerable plaque by either imaging or laboratory methods or both is very important for early intervention to minimize the burden of cardiovascular complications as stroke or myocardial infarction hence vulnerable plaque is responsible for the majority cardiovascular events. Serum neopterin is a good marker of plaque vulnerability both in carotid and coronary arteries.

Serum neopterin is a specific marker of macrophages activation which play a central role in the pathogenesis of atherosclerosis.

Serum neopterin is a valuable marker of redox system activation reflecting the potential effect of reactive oxygen species (ROS) which play a role in endothelial dysfunction and diabetic micro and macro vascular complications.

Serum neopterin reflects innate immunity system activation more specifically than the non specific Hs CRP as an inflammatory marker while assessing and managing patients with cardiovascular diseas 


\section{References}

Abdushi SA, Nazreku FD, Ukë Kryeziu F. (2013) : Increased carotid intima-media thickness associated with high hs-CRP levels is a predictor of unstable coronary artery disease. Cardiovascular Journal of Africa; 24(7):270-273.

Ammirati E, Moroni F, Norata GD, et al. (2015): Markers of Inflammation Associated with Plaque Progression and Instability in Patients with Carotid

Atherosclerosis. Mediators of Inflammation; 2015:718329.

\section{Bjørnestad EØ, Borsholm RA, Svingen} GFT, et al. (2017): Neopterin as an Effect Modifier of the Cardiovascular Risk Predicted by Total Homocysteine: A Prospective 2-Cohort Study. Journal of the American Heart Association: Cardiovascular and Cerebrovascular Disease; 6(11):e006500.

Elayalwar S, Lakshmipathi J.(2016): A study to assess the association between serum neopterin levels and various biochemical, clinical, and echocardiographic variables. J. Evolution Med. Dent. Sci ;5(57):3890-3893

Fuchs D, Weiss G, Wachter H. (1993): Neopterin, biochemistry and clinical use as a marker for cellular immune reactions. Int Arch Allergy Immunol; 101:1-6.

Gieseg SP, Crone EM, Flavall EA, et al. (2008): Potential to inhibit growth of atherosclerotic plaque development through modulation of macrophage neopterin/7,8dihydroneopterin synthesis. Br J Pharmacol; 153:627-35.
Kalofoutis C, Piperi C, Kalofoutis A, et al. (2007). Type II diabetes mellitus and cardiovascular risk factors: Current therapeutic approaches. Exp Clin Cardiol; 12:17-28.

Kojima S, Icho T, Kajiwara Y, et al.(1992): Neopterin as an endogenous antioxidant. FEBS Lett. ; 304:163-166.

Larsen M, Bayard C, Lepetitcorps H, et al. (2017): Elevated Neopterin Levels Predict Early Death in Older Hip-fracture Patients. EBioMedicine; 26:157-164.

Kablak-Ziembicka A , Przewlocki T, Sokołowski A, et al. (2011):

Atherosclerosis 214 : 185-190

Liao H, Hong H, Wang H (2015): Serum Neopterin as a Novel Marker for Carotid Artery Stenosis in Community Subjects. J Neuro Cardiovasc Dis2: 009.

Lin HS, Tsai TH, Liu CF, et al. (2012): Serum level and prognostic value of neopterin in patients after ischemic stroke. Clin Biochem; 45:1596-601

Liu ZY, Li YD. (2013): Relationship between serum neopterin levels and coronary heart disease. Genet Mol Res; 12(4):4222-9.

Murr C, Grammer TB, Meinitzer A, et al. (2014): Immune activation and inflammation in patients with cardiovascular disease are associated with higher phenylalanine to tyrosine ratios: the Ludwigshafen Risk and Cardiovascular Health Study. J Amino Acids; 2014:783730. Orasanu G, Plutzky J (2009): The pathologic continuum of diabetic vascular disease. J Am Coll Cardi ol; 535 Suppl: S35-42. Packard RR, Libby P (2008):

Inflammation in atherosclerosis: from 
vascular biology to biomarker discovery and risk prediction. Clin Chem 54: 24-38.

Parildar H, Gulmez O, Cigerli O, et

al.(2013): Carotid artery intima media thickness and HsCRP: Predictors for atherosclerosis in prediabetic patients. Pak J Med Sci; 29(2):495-499. doi:

http://dx.doi.org/10.12669/pjms.292.3133

Shirai R, Sato K, Yamashita T, et al. (2018): Neopterin Counters Vascular Inflammation and Atherosclerosis. Journal of the American Heart Association: Cardiovascular and Cerebrovascular Disease ;7(3):e007359.

\section{Sugioka K, Naruko T, Hozumi T, et} al.(2010): Elevated levels of neopterin are associated with carotid plaques with complex morphology in patients with stable angina pectoris. Atherosclerosis; 208:52430 .

van Dijk RA, Rijs K, Wezel A, et al. (2016): Systematic evaluation of the cellular innate immune response during the process of human atherosclerosis. J Am Heart Assoc; 5:e002860.

\section{Zeng X, Zhang G, Yang B, et al.(2016):}

Neopterin as a Predictor of Functional

Outcome and Mortality in Chinese Patients with Acute Ischemic Stroke. Mol Neurobiol; 53(6):3939-3947. 